\title{
The Importance of Brain MRI in the Diagnosis of Marchiafava-Bignami Disease
}

\author{
Pontes $\mathrm{CM}^{*}$, Cagy M, Santos R and Soares CN
}

Neurology Department, Hospital Federal dos Servidores do Estado, Rio de Janeiro, Brazil

*Corresponding author: Pontes CM, Neurology Department, Hospital Federal dos Servidores do Estado, Rua Sacadura Cabral no 178, Saúde. Rio de Janeiro, Cep: 20221-903, Brazil, E-mail: caio.m88@gmail.com

Citation: Pontes CM, Cagy M, Santos R, Soares CN (2017) The importance of brain MRI in the diagnosis of Marchiafava-Bignami disease. J Neurol Neurol Disord 3(2): 201. doi: 10.15744/2454-4981.3.201

Received Date: March 06, 2017 Accepted Date: June 02, 2017 Published Date: June 05, 2017

\begin{abstract}
Marchiafava-Bignami disease (MBD) is a neurological disorder that has been found to be associated with chronic alcoholism and malnutrition. We report a 45 year old man, chronic alcoholic that developed discouragement for activities involving daily living, changes in retrograde memory in addition to mutism and gait instability. Brain MRI showed central atrophy of the corpus callosum(CC), hypointensity(necrosis) and ventricular dilation(white matter and subcortical region involvement). Pathological characteristics include isolated demyelination and axonal loss in the central layer of the CC sparing the dorsal and ventral layer. This pattern of necrosis of the middle layer of the CCis a typical finding in the disease. The clinical diagnosis has considerably changed during recent decades after brain MRI provided the opportunity of a reliable in-vivo diagnosis. With early detection and treatment, the prognosis of MBD may be good.
\end{abstract}

Keywords: Marchiafava-Bignami disease; Corpus callosum; Cronic alcoholic

\section{Introduction}

Marchiafava-Bignami disease (MBD) is a rare complication related to alcohol abuse and chronic malnutrition which results in demyelination and symmetrical necrosis of the central layer of the corpus callosum [1]. Serum anion gap and osmotic gap can be observed in patients ingesting etanol. The serum anion gap still might be high, which could lead to the demyelination syndrome. The increase in serum osmolarity produced by alcohol is relied onserum concentration, molecular weights, and metabolic rate. Toxic effects of alcohol and its metabolites can cause demyelination of the corpus callosum [2]. Despite alcohol is the principal element, there are other causes that can be factors such as cyanide and CO poisoning, sepsis, sickle cell disease and Plasmodium infection [3]. The clinical presentation is variable, including mental confusion, dementia, psychosis, spasticity and dysarthria [4]. Since the symptoms are not specific, the clinical diagnosis can be difficult, being the magnetic resonance imaging (MRI) of pivotal value in the investigation [5]. Diffuse thickening of the CC andT1 and T2 prolonged areas in the CC are considered characteristic MRI findings at the acute phase. It has been reported that the first change on MRI is diffuse swelling of the corpus callosum, followed by a genu lesion, and finally by a splenium lesion, sparing the rostrum [5,6]. T1-weighted MRI in the subacute to chronic stage shows hypointense cystic-necrotic lesions in the corpus callosum in addition to callosal atrophy. Extracallosal lesions have been reported involving predominantly the periventricular white matter or the basal ganglia [7].

\section{Case report}

Man, 45 years old, bus driver and chronic alcoholic. According to his wife's report, in April 2014 he developed changes in executive skills, inattention, gait instability and alteration in memory for spatial layouts(ability to recognize environments learned long time ago). Progressive worsening occurred with dysarthria which resulted in mutism after 6 months. On examination there was gait instability. During hospitalization he performed blood tests such as VDRL, TPHA, anti-HIV 1 and 2, anti-HTLV 1 and 2, hepatitis B and C, vitamin B12, all with values normal. Magnetic resonance imaging of the skull demonstrated atrophy of central portion of the corpus callosum and focus of hypointensity compatible with necrosis, in addition to ventricular dilation. The patient was treated with vitamin B1 (300 mg/day) and vitamin B12 (5000 mcg/day) resulting in a great improvement in mutism, gait instability and inattention. There had been partial resolution of radiological signs after six months Figure 1a-1d. 


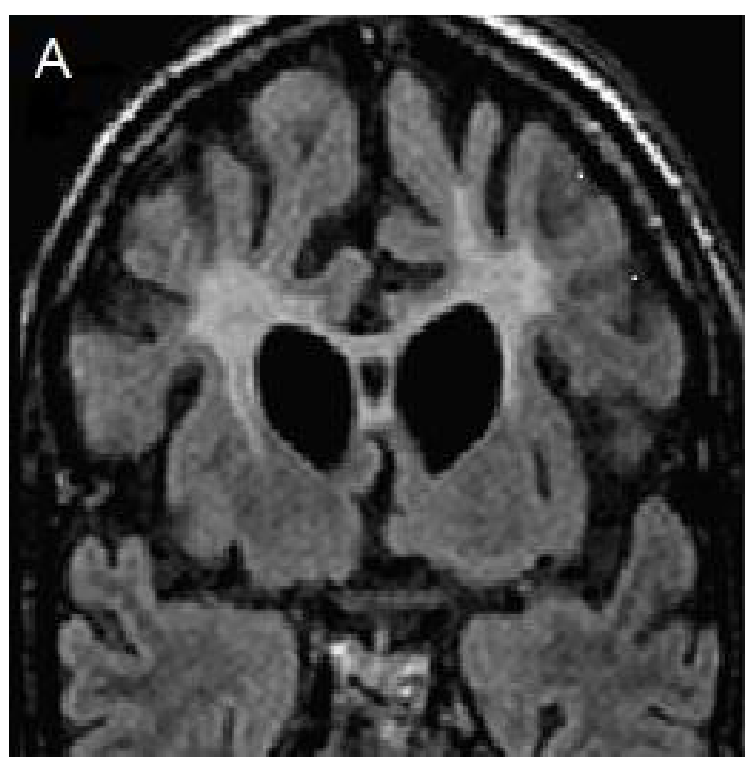

Figure 1a: Coronal flair showing demyelination of corpus callosum and commitment of periventricular white matter Sagital

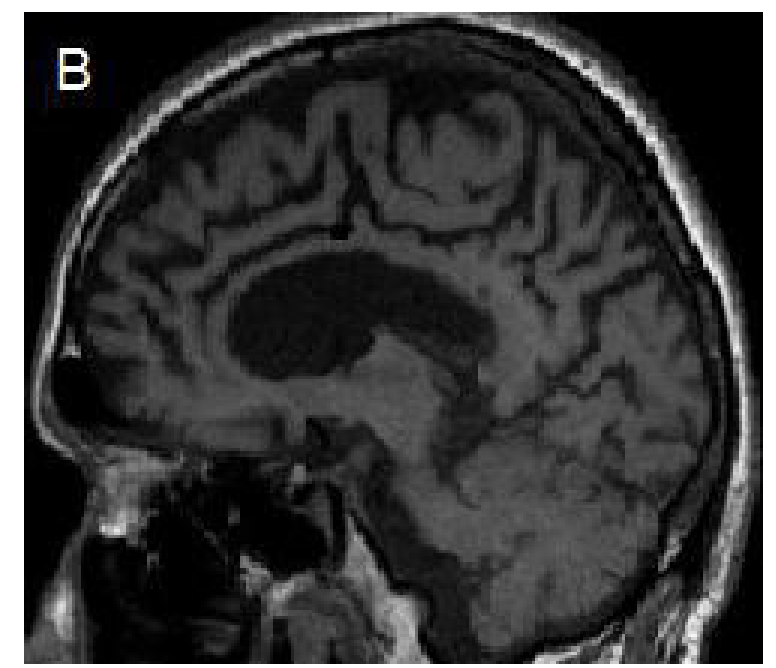

Figure 1b: Sagital T1 showing necrosis in splenium of corpus callosum

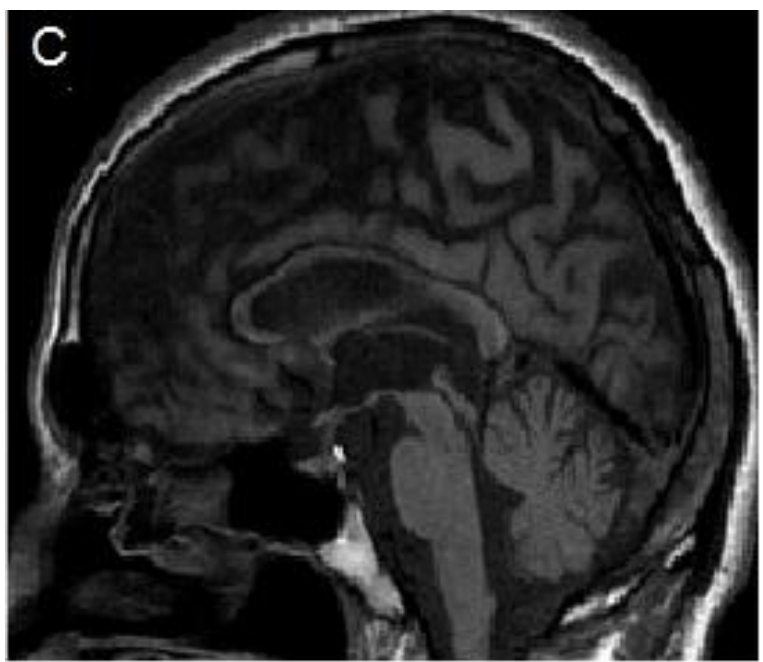

Figure 1c: Sagital T1 showing diffuse tapering of corpus callosum("Sandwich sign") 


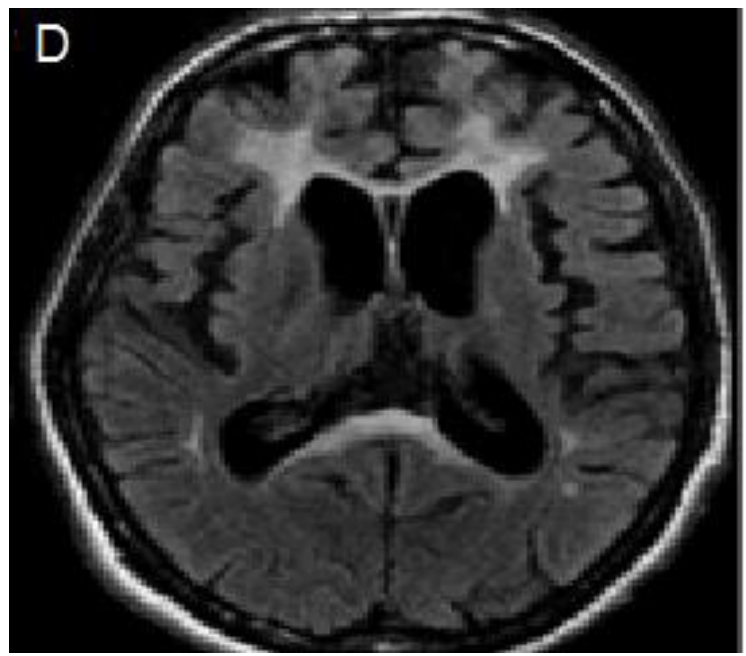

Figure 1d: Axial flair showing periventricularcommitment of genu and splenium("Boomerang sign") in addition to cerebral atrophy

\section{Discussion}

MDB was described in 1903 by two Italian neuropathologists, Ettore Machiafava and Amico Bignami who observed three chronic alcoholics with symptoms of psychomotor agitation, convulsions, and declining level of consciousness $[1,8]$. All patients died after having seizures and coma. In each one, the middle two thirds of the corpus callosum was found to be severely necrotic. Neuropathological studies have revealed demyelination and corpus callosum atrophy [9]. Until recently, the definitive diagnosis of MBD could only be confirmed at necropsy. However, with the advent of neuroimaging, it was possible to define it through the previous history of alcoholism and pathological changes in the corpus callosum, demonstrated by means of MRI [10]. On the past (1977) Brion et al had introduced a classification for MBD categorizing an acute, a subacute, and a chronic form [5].

- Acute state - seizures, alterations of consciousness, and death may occur.

- Subacute state - characterized by mental confusion, behavioral disorders, memory deficits, cerebellar signs and interhemispheric disconnection.

- Chronic state - mild dementia.

Whereas the above mentioned clinical classification is still widely used, Heinrich et al proposed a clinicoradiologic classification considering the patients's clinical status as well as the radiological severity of cerebral changes detectable in MRI. In 2004 they stressed, that the terms "acute,subacute and chronic" should be abandoned to describe a subtype of MBD. Cases can be assigned to one of two groups, which they refer to as type A or type B, based on a review of 50 radiologic cases diagnosed in vivo:

Type A - Predominant features of coma and stupor, seizures, limb hypertonia; this subtype is associated with a high prevalence of pyramidal tract symptoms. Radiologic features include involvement of the entire corpus callosum.

Type B - Normal or mildly impaired mental status, rapid onset of dementia, limb hypertonia, dysarthria and astasia-abasia. It was considered typical for subacute forms. Radiologic features are partial or focal callosal lesions. Type B has favourable prognosis and lesions may reverse suggesting an underlying edema rather than demyelination [7].

At present, the diagnosis is made on the basis of clinical findings in combination with radiological imaging features [11] Table 1.

\begin{tabular}{|c|c|}
\hline MRI findings & Underlying pathophysiology \\
\hline Hyperintensity on T2 weighted images & Edema and myelin damage \\
\hline Hypointensity on T1 weighted images & $\begin{array}{c}\text { Total loss of myelin with replacement of the } \\
\text { region by a cyst }\end{array}$ \\
\hline $\begin{array}{c}\text { Hyperintense rims and hypointense cores on FLAIR } \\
\text { images }\end{array}$ & $\begin{array}{c}\text { Damage to the myelin at the rim with a } \\
\text { central necrotic area }\end{array}$ \\
\hline Uniformly hyperintense lesions on FLAIR & Mixture of demyelination and edema \\
\hline Areas of restricted diffusion on DWI (acute phase) & Cytotoxic edema \\
\hline
\end{tabular}

Table 1: Correlation of imaging findings in MBD with pathophysiology

The corpus callosum is the largest commissural white matter bundle in the brain containing 200-250 million interhemispheric fibers [12,13]. Major portion of the corpus callosum receives its arterial supply from the carotid system except for splenium, which is supplied by the vertebrobasilar system [14]. MRI is currently the most sensitive diagnostic tool as well as to monitor the evolution toward partial or total regression $[11,15]$. Acute or subacute lesions are characterized by edema and early myelin damage. The corpus callosum lesions are hypo-intense on T1-weighted image and hyperintense on T2-weighted/fluid-attenuated 
inversion recovery (FLAIR) images. Fast spin-echo and T2-weighted MRI scans show hyperintensity with nonenhancing on postgadolinium images [8]. During the subacute phase, cystic lesions begin to develop. In chronic stage hypointensity on T1weighted images is mainly related to a total loss of myelin, with necrosis, gliosis and replacement of the region by a cyst and residual atrophy of the involved structures [15]. FLAIR images may show central hypointense signal and peripheral hyperintense rim ("Sandwich sign") [8]. Another signal that can be observed is hiperintensity involving the entire splenium ("Boomerang sign") [12]. Although the callosal lesions have been described as the hallmark of this disease, few cases of MBD also demonstrate signal intensity abnormalities in frontal lateral and temporal cortices (Morel's cortical laminar sclerosis) [16]. Other imaging modalities include MR spectroscopy (MRS) which shows increase in choline and increased choline/creatine (Cho/ Cr) ratio in acute phase. Lactate peak is usually seen in the acute/subacute phase of demyelination. SPECT studies show bilateral cortical and/ or subcortical hypoperfusion. This perfusion defected notes that the disease doesn't only affect corpus callosum $[12,17,18]$. The aim of serial MR imaging and MR spectroscopy in Marchiafava-Bignami disease is to note changes after vitamin therapy and to assess the contribution to in vivo study of the disease[16].

Differential diagnosis includes infarction of recurrent artery of Heubner, neoplastic disease such as astrocytoma or lymphoma of corpus callosus, demyelinating disease such as multiple sclerosis (MS), Wernicke encephalopathy, progressive multifocal leukoencephalopathy, HIV related encephalopathy, neurosyphilis and acute disseminated encephalomyelitis [19].

Strict alcohol abstinence with counseling is the best core of the treatment program. Pharmacological treatment consists of administration of thiamine IV $500 \mathrm{mg}$ three times a day (TID) for 3 days and then $100 \mathrm{mg}$ per os (PO) [20]. Also clinical improvement could be observed using high dose of corticosteroids(250 mg methyl-prednisolone ), others B-complex vitamins, folic acid and amantadine (100mg $2 x$ per day) [21-23]. The available evidence suggests that an effective and aggressive early treatment is often associated with marked clinical improvement [24].

\section{Conclusion}

Advances in neuroimaging have made early diagnosis of MBD possible and help in early initiation of treatment. Clinical evidences support that clinical improvement of patients with MBD is associated with radiological improvement as well. Fast and nearcomplete relieve of symptoms is possible, even though involvement of the entire corpus callosum and the cortex. MBD may be a reversible brain disease.

\section{References}

1. Marchiafava E, Bignami A (1903) Sopra un alterazione del corpo calloso osservata in soggetti alcoolisti. Riv Patol Nerv Ment 8: 544-9.

2. Xiaodong Chen, Yuanwei Wang, Ying Wang, Daoming Tong, Baolin Zhu (2016) Alcoholic and Non-Alcoholic Marchiafava-Bignami Disease: A Comparative Study. Neurosci Med 7: 99-105.

3. Yongjian Cui, Lei Zheng, Xiaoli Wang, Weiwen Zhang, Dongcai Yuang and Yan Wei (2015) Marchiafava-Bignami disease with rare etiology: A case report. Exp Ther Med 9: 1515-7.

4. Carla Gramaglia, Alessandro Feggi, Camilla Vecchi, Sarah Di Marco, Alessandra Venesia et al (2016) Marchiafava-Bignami Disease with frontal cortex involvement and late onset, long-lasting psychiatric symptoms: a case report. Riv Psichiatr 51: 79-82.

5. Holger Wenz, Philipp Eisele, Dimitrios Artemis, Alex Forster, Marc A Brockmann (2014) Acute Marchiafava-Bignami Disease with Extensive Diffusion Restriction and Early Recovery: Case Report and Review of the Literature. J Neuroimaging 24: 421-4.

6. Aggunlu L, Oner Y, Kocer B, Akpek S (2007) The value of diffusion weighted imaging in the diagnosis of Marchiafava-Bignami disease: a propos of a case. J Neuroimaging 18: 188-90.

7. Alexander Heinrich, Uwe Runge, Alexander V Khaw (2004) Clinicoradiologic subtypes of Marchiafava-Bignami disease. J Neurol 251: 1050-9.

8. Shahina Bano, Shibani Mehra, Sacchida Nand Yadav, Vikas Chaudhary (2009) Marchiafava-Bignami disease: Role of neuroimaging in the diagnosis and management of acute disease. Neurol India 57: 649-52.

9. Sunil Kumar K, Rimiki Challam, Naveen J, W. Jatishwor Singh (2014) Marchiafava - Bignami Disease: A Case Report. J Clin Diagn Res 8: RD01-2.

10. Matti Hillbom, Pertti Saloheimo, Shinsuke Fujioka, Zbigniew K Wszolek, Seppo Juvela et al (2014) Diagnosis and management of Marchiafava-Bignami disease: a review of CT/MRI confirmed cases. J Neurol Neurosurg Psychiatry 85: 168-73.

11. Kathyayini Paidipati Gopalkishna Murthy (2014) Magnetic Resonance Imaging in Marchiafava-Bignami Syndrome: A Cornerstone in Diagnosis and Prognosis. Case Rep Radio 2014: ID 609708.

12. Hardeep Singh Malhotra, Ravindra Kumar Garg, Mukund R Vidhate, Pawan Kumar Sharma (2012) Boomerang sign: Clinical significance of transient lesion in splenium of corpus callosum. Ann Indian Acad Neurol 15: 151-7.

13. Christopher G Filippi, Keith A Cauley (2014) Lesions of the Corpus Callosum and Other Commissural Fibers: Diffusion Tensor Studies. Semin Ultrasound CT MR 35: 445-58.

14. Fitsiori A, Nguyen D, Karentzos A, Delavelle J, Vargas MI (2011) The corpus callosum: White matter or terra incognita. Br J Radiol 84:5-18.

15. Tekwani Parmanand H (2016) Marchiafava-Bignami disease in chronic alcoholic patient. Radiol Case Rep 11: 234-7.

16. Gambini A, Falini A, Moiola L, Comi G, Scotti G (2003) Marchiafava-Bignami Disease: Longitudinal MR Imaging and MR Spectroscopy Study. Am J Neuroradiol 24: 249-53.

17. Yahya Celik, Meryem Kaya, Semra Sengün, Ufuk Utku (2002) Marchiafava-Bignami disease: cranial MRI and SPECT findings. Clin Neurol Neurosurg 104: $339-41$.

18. Rudkin TM, Arnold DL (1999) Proton magnetic resonance spectroscopy for the diagnosis and management of cerebral disorders. Arch neurol 56: 919-26.

19. Chang N, Kar-Wai E, Jung S, Liao C (2010) A Rare Case of Marchiafava-Bignami Disease in Taiwan. J Radiol 35: 185-90. 
20. M Tong, V Laskova (2015) Marchiafava-Bignami disease: A case report with good prognosis. J Inter Med 30: s423.

21. Kikkawa Y, Takaya Y, Niwa N (2000) A case of Marchiafava-Bignami disease that responded to high-dose intravenous corticosteroid administration. Rinsho Shinkeigaku. 40 : $\quad 1122-5$.

22. Ault Jennifer, Tarakad S Ramachandran (2016) Marchiafava-Bignami Disease Clinical Presentation.

23. Staszewski J, Macek K, Stepien A (2006) Reversible demyelinisation of corpus callosum in the course of Marchiafava-Bignami disease. Neurol Neurochir Pol 40: 156-61.

24. Debasish Mishra, Priya Bhate, Vishal Anand Gupta, Shilpa Sankhe et al. (2016) Altered Sensorium in a Young Male: Marchiafava BignamiDisease Revisited. J Asso Physicians India 64: 86-8.

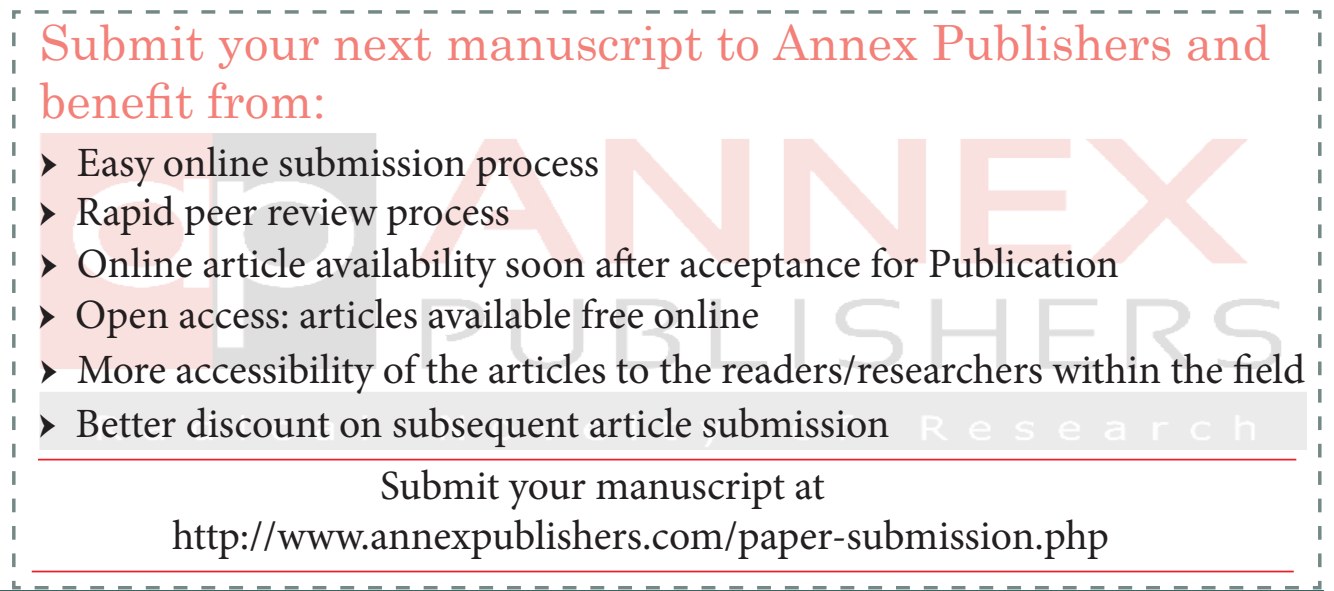

